\title{
Percutaneous Left Atrium Appendage Closure as an Option for Systemic Anticoagulation: Success and Safety Outcomes by a Review Series Analysis
}

\author{
Jonathan Powell* and Francisco Nascimento \\ Department of Medicine, Florida Atlantic University, USA
}

Submission: June 06, 2017; Published: July 05, 2017

*Corresponding author: Jonathan Powell, Department of Medicine, Florida Atlantic University, USA, Email: powellj@health.fau.edu

\begin{abstract}
Objective: To perform a review series about percutaneous LAA closure, evaluating the initial case series and their success and safety outcomes.

Methods: A systematic review with standard protocols recommended by PRISMA was performed. We reviewed relevant studies and the absolute numbers of events for each outcome of interest in every study were obtained. RevMan statistical software program was used to synthesize the results. Dichotomous outcomes data were measured and reported as the odds ratio (OR) with their $95 \%$ confidence intervals. Heterogeneity across studies was examined with the Cochrane's Q statistic (based on pooled OR) and the I ${ }^{2}$ statistic test.

Results: Seven studies were included in this analysis, culminating to 1215 patients in the device closure group. The primary outcome studied was ischemic stroke incidence. 31 ischemic stroke events occurred in the device closure arm, versus 27 events in the control arm, risk ratio of $0.7095 \% \mathrm{CI}[0.40,1.21]$ the primary endpoint was found to be non-inferior in the device arm compared to the control arm with a probability of non inferiority of $>0.999$. More hemorrhagic strokes occurred in the medical management group, $7 / 532$ compared to $5 / 1215$ in the device cohort. Regarding device safety, the overall incidence of procedure-related complications was low, with most outcomes occurring approximately $1-2 \%$ of the time. The most common complication of LAA closure device placement was pericardial effusion.
\end{abstract}

Conclusions: The transcutaneous LAA closure using an occlusion device is efficient to prevent stroke and TIA and safe when performed by experienced operators.

\section{Introduction}

As the overall population is aging and living longer, atrial fibrillation (AF) becomes more prevalent. In 2010 it was calculated that 5 million Americans had AF, and the United States of America (USA) is expected to have 12 million AF patients by 2030 [1]. Stroke remains a significant cause of morbidity and mortality worldwide with annual incidence of approximately 800,000 in the USA alone, with $80 \%$ of those due to focal ischemia originated from arterial occlusion [2]. Oral anticoagulation (OAC) has been used as the main treatment of $\mathrm{AF}$ for stroke prevention, with warfarin as the only regimen as options were lacking. During the recent past years OAC alternatives have emerged, and nowadays some new options are available with at least the same efficacy and safety profile, more reliable anticoagulation level, and without the need of frequent monitoring [3-6]. Despite all pharmacological evolution, 30 to $50 \%$ of AF patients do not receive chronic anticoagulation, due to relative or absolute contraindications, or patient or physician barriers, including the fear of treatment-induced bleeding, thus limiting the use of OAC $[7,8]$. Device based therapies are currently being developed for stroke prevention in AF to potentially offer an alternative approach to this problem [9].

It is assumed that $90 \%$ of the clinically apparent embolism in $\mathrm{AF}$ originates from the left atrium appendage (LAA) [10]; therefore, it would be reasonable to occlude the LAA as an alternative treatment to OAC. Since the first-in-man percutaneous LAA closure was performed in 2001 with the PLAATO device (PLAATO System, ev3 Inc., Plymouth, Minnesota) [11], interest in percutaneous LAA intervention has grown and there are currently several different occlusion devices being developed for this purpose. Although the PLAATO device has not been approved for clinical use, the dedicated Watchman device (Boston Scientific-Atritech, Minneapolis, Minnesota) is 
approved in the United States and Europe, and the Amplatzer Cardiac Plug [ACP] (St. Jude Medical Inc., Saint Paul, Minnesota) device is approved in Europe. However, randomized clinical trials (RCT) are sparse regarding this procedure, and case-series and registry data have generated some controversy and concerns amongst different specialists and regulatory agencies. The safety of the procedure has been a major point of discussion, as LAA closure is known to be a challenging procedure which confers a steep learning curve for the operators. The recently published PROTECT AF trial [12] is currently the only randomized trial to evaluate the safety of this technology. We aim to perform a review series about percutaneous LAA closure, evaluating the initial case series and their success and safety outcomes.

\section{Methods}

We performed this review with standard protocols recommended by the Preferred Reporting Items for Systematic reviews and meta-analysis (PRISMA) [13]. The search for pertinent articles was performed in PubMed, Medline and Cochrane database in August 2016 using the key words and phrases as "leftatrium appendage closure”, "leftatrium appendage occlusion", "left atrium appendage closure and atrial fibrillation", "left atrium appendage closure and stroke", "percutaneous left atrium appendage closure", "PLAATO", "Watchman", "Amplatzer Cardiac Plug" .There was no date or language restriction for our selection of publication. Abstracts from international cardiology meeting programs (American College of cardiology, American Heart Association, European Society of Cardiology, Transcatheter Cardiovascular Therapeutics, and Society for Cardiac Angiography and Interventions) were also searched for relevant data. We performed literature searches, and individually reviewed relevant articles and extracted data independently. Discrepancies between study design and obtained data were resolved by consensus amongst the authors if necessary.

\section{Inclusion criteria}

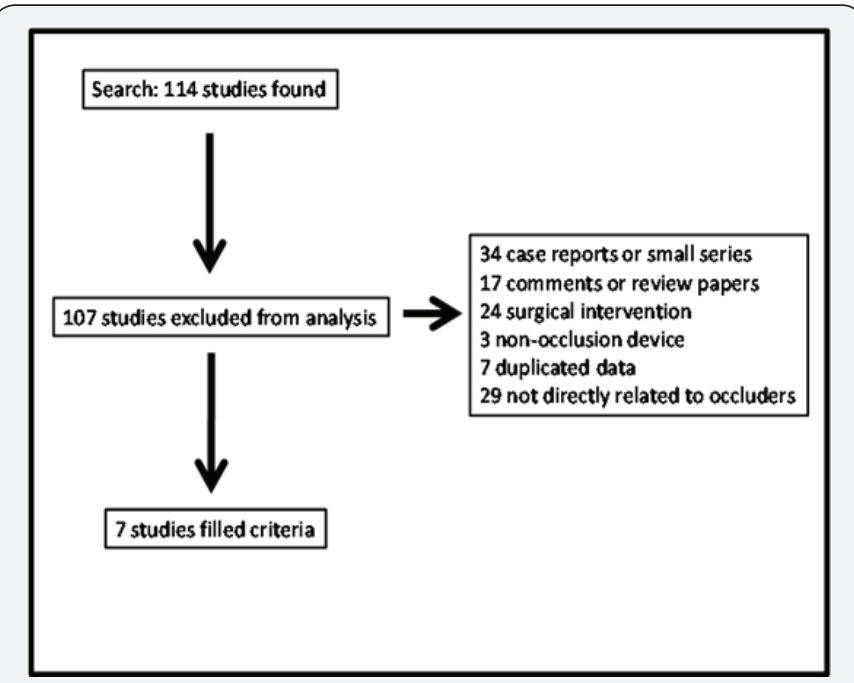

Figure 1: Flow chart of included studies for analysis.
For the purpose of this review, studies were selected to be analyzed if all the following inclusion criteria were met:
A. The method of LAA exclusion by percutaneous occlusion device only.

B. The RCT, registries or case series with at least 50 cases described to avoid low volume centers bias.

C. The CHADS2 score being reported.

D. The complications being reported.

E. The study has been published. Exclusion criteria were: studies without access to full data or manuscript. Total of 7 studies met criteria for analysis (Figure 1).

\section{Study cohort}

We evaluated data pertaining to all patients presented in the case series or registries who underwent LAA closure, as well as all patients enrolled in the randomized controlled trial PROTECT AF that underwent LAA closure or medical therapy alone. For the purpose of comparison of stroke events, the studies where no control arm has been used, the correspondent CHADS2 score for that cohort was used to estimate stroke risk/year and then compared.

\section{Study endpoints}

The primary outcome for this review series was a composite of recurrent nonfatal CVA and TIA. We also analyzed each component of the primary outcome individually as secondary end points. There was no standardized definition of safety parameters across all studies included herein. Attention was given to report adverse events related to the procedure or to the devices.

\section{Description of the Left Atrium Appendage and Devices used}

\section{Left atrium appendage}

The LAA is a small, muscular sacculation of the left atrium (LA) located between the left upper pulmonary vein and the left ventricle, overlapping the proximal left circumflex artery. It is a derivative of the primitive atrium and has a wide anatomic variability. Its size ranges from 20 to $45 \mathrm{~mm}$ in length and 15 to $35 \mathrm{~mm}$ in orifice diameter. In more than two-thirds of the population the LAA consists of two or more lobes originating from one common orifice [14].

\section{Amplatzer cardiac plug}

The ACP is a self-expandable nitinol device with a polyester patch within, formed by three parts: a cylindrical lobe with a fixed length of $6.5 \mathrm{~mm}$, to which diameter $(16-30 \mathrm{~mm}$, stepwise by $2 \mathrm{~mm}$ ) the prosthesis size refers; an occlusive disc, $4 \mathrm{~mm}$ larger than the $16-22 \mathrm{~mm}$ prosthesis, and $6 \mathrm{~mm}$ larger than the 24-30mm devices; and a flexible central connector pine. There are six pairs of barbs attached to the lobe and directed to the 
disc, all identified by radio paque marks, to enhance retention of the lobe in the LAA (Figure 2).

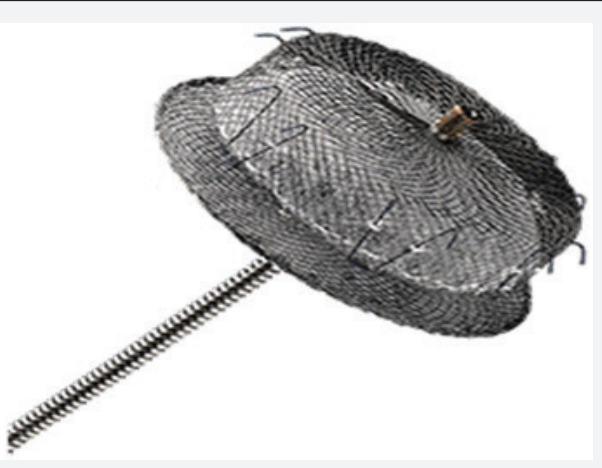

Figure 2: Amplatzer Cardiac Plug (ACP) device.

\section{PLAATO}

The PLAATO device consists of a self-expanding nitinol cage (range of diameter 15 to $32 \mathrm{~mm}$ ) covered with expanded polytetra fluoro ethylene (PTFE) in order to close off blood flow into the remaining part of the LAA. Three rows of anchors along the struts help stabilize the occluder in the appendage (Figure $3)$.

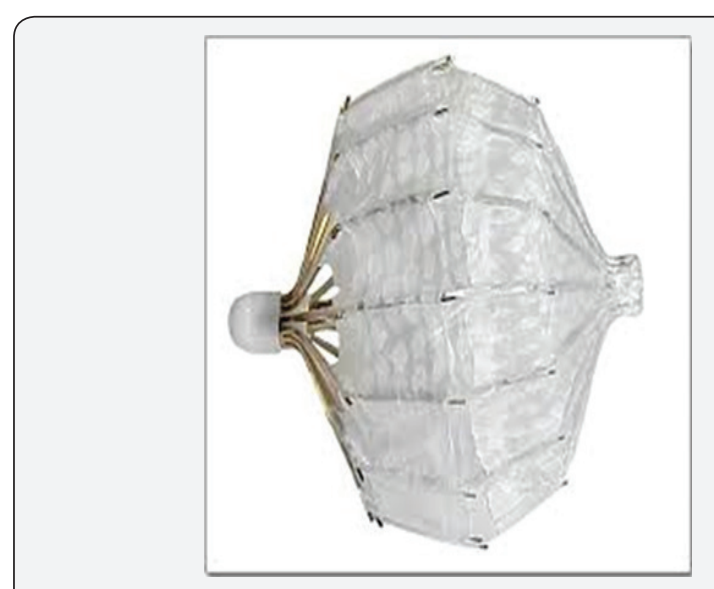

Figure 3: Plaato device.

\section{Watchman}

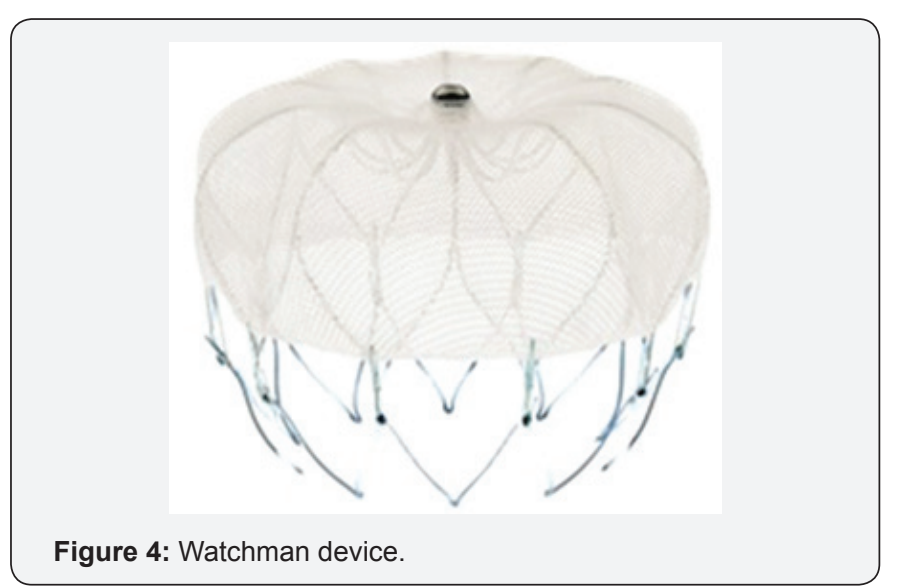

The Watchman device is a parachute-shaped device with a self-expanding nitinol frame structure and a permeable polyester membrane over the atrial side with mid-perimeter fixation bards to secure it in the LAA. The device is available in diameters ranging from $21 \mathrm{~mm}$ to $33 \mathrm{~mm}$ to accommodate the variability in LAA width (Figure 4).

\section{Statistical analysis}

Information on study design, sample size, demographic characteristics, outcome, and follow-up data were extracted and entered into a data sheet using a standardized protocol. The absolute numbers of events for each outcome of interest in every study were extracted. The Cochrane Collaboration's Review Manager (RevMan) statistical software program was used to synthesize the results. The Mantel-Haenszel method was used to account for the differences between studies. Dichotomous outcomes data were measured and reported the odds ratio (OR) with their 95\% confidence intervals. Heterogeneity across studies was examined with the Cochrane's Q statistic (based on pooled OR) and the $\mathrm{I}^{2}$ statistic test. An $\mathrm{I}^{2}$ value of $<25 \%$ was considered low heterogeneity and $>50 \%$ was considered significant heterogeneity. The weight of each trial on the overall results of meta-analysis outcome was calculated as a percentage of the number of patients in that given trial over the total number included in each outcome analysis.

\section{Results}

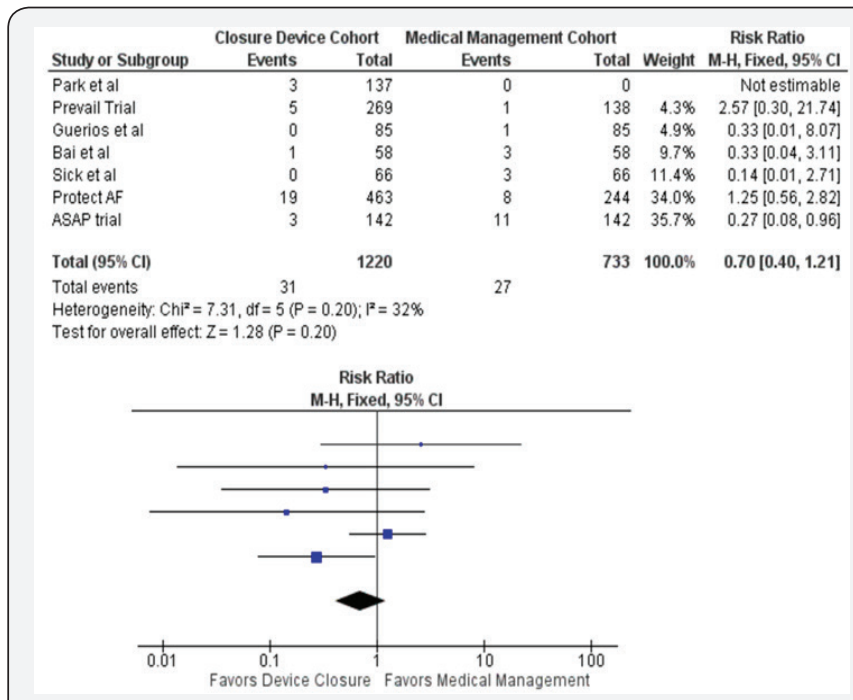

Figure 5: Ischemic stroke incident rate- device based laa closure vs medical management.

Seven studies were included in this analysis, culminating to 1215 patients in the device closure group. The primary outcome studied was the ischemic stroke incidence rate in the device closure group compared to the control. For the purpose of comparison of ischemic stroke events, the studies where no control arm was used the corresponding CHADS VASC score for that cohort was used to estimate stroke risk/year and 
then compared. A total of 31 ischemic stroke events occurred in the device closure arm (1215 patients), versus 27 events in the control arm (741 patients), with a risk ratio of 0.70 $95 \%$ CI $[0.40,1.21]$ as illustrated by Figure 5 . The risk ratio criterion (treatment over control) of 1.75 was used to establish noninferiority (Table 1). The null hypothesis would be rejected

Table 1: Included studies in the analysis. if the risk ratio between rates in the randomized groups satisfied the noninferiority criteria. Therefore, the primary endpoint was found to be non-inferior in the device arm compared to the control arm with a probability of non inferiority of $>0.999$ (Figure 5).

\begin{tabular}{|c|c|c|c|c|c|c|}
\hline & Devices & Patients ITT & Patient AT & Control Group & $\begin{array}{c}\text { Avg CHADS VASC } \\
\text { Score }\end{array}$ & $\begin{array}{c}\text { Mean Follow up } \\
\text { Period }\end{array}$ \\
\hline $\begin{array}{c}\text { Reddy et al. [13] } \\
\text { Protect AF }\end{array}$ & Watchman & 463 & 463 & 244 & $2.20 \%$ & $27.6 \pm 13.2$ months \\
\hline $\begin{array}{c}\text { Reddy et al. [13] } \\
\text { ASAP }\end{array}$ & Watchman & 150 & 142 & NA & $4.4 \% \pm 1.7 \%$ & $14.4 \pm 8.6$ months \\
\hline Guerios 2012 & ACP & 86 & 85 & NA & $3.6 \% \pm 1.6$ & 3.6 months \\
\hline Bai 2012 & Watchman & 58 & 58 & NA & $2.2 \pm 1.0$ & $25.9 \pm 13.4$ months \\
\hline Park 2011 & ACP & 137 & 137 & NA & $\begin{array}{c}\text { not applicable } \\
\text { (retrospective } \\
\text { preregistry data } \\
\text { collection) }\end{array}$ \\
\hline Prevail 2014 & PLAAT0 & 180 & 162 & 138 & $3.8 \pm 1.2$ & $11.8 \pm 5.8$ months \\
\hline Sick 2007 & Watchman & 66 & 66 & NA & $1.8 \pm 1.1$ & 24 months \\
\hline
\end{tabular}

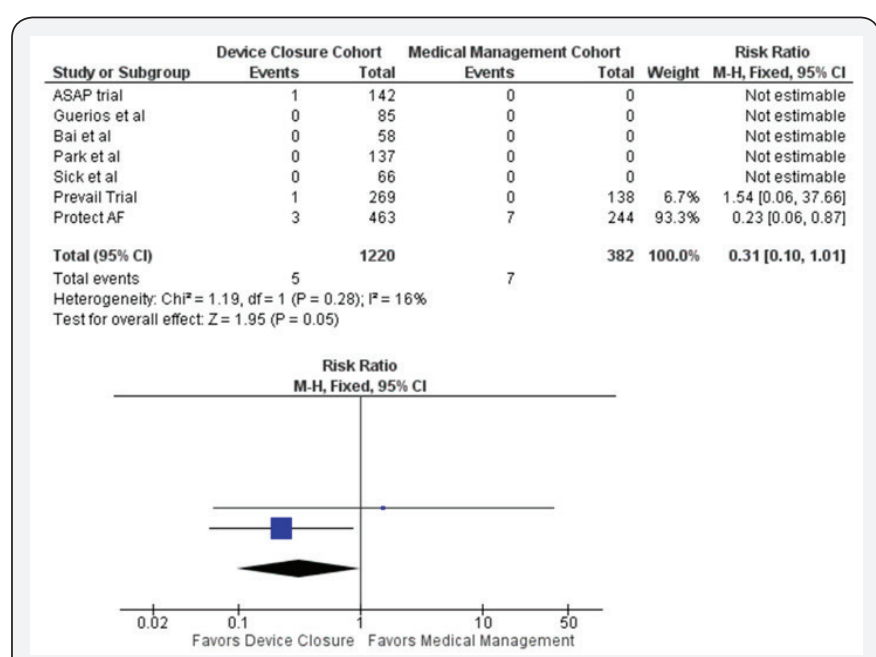

Figure 6: Hemorrhagic stroke incident rate- device based laa closure vs medical management.

The incidence of hemorrhagic strokes among both cohorts was rare relative to ischemic strokes. A larger proportion of hemorrhagic strokes occurred in the medical management group, 7 patients of 532 compared to 5 of 1215 in the device cohort. This is most likely attributable to prolonged use of systemic anticoagulation in this group as opposed to short term (avg. of 3 months) in the device closure group. Although the incidence of hemorrhagic stroke was lower for the device based therapy group, this did not quite achieve statistical significance, with a risk ratio of $0.3195 \%$ CI $[0.10,1.01]$ as demonstrated in (Figure 6). A confounding factor that contributed to this lack of statistical significance was the lack of a control group in most of the studies within this analysis. Only PREVAIL and PROTECT AF were designed with a control group; thus, the power of the results for this endpoint is too low to achieve statistical significance. Although one could utilize the HASBLED scoring system to estimate the bleeding occurrence rate in the populations studied in the other trials, those values would be speculative and appropriate for hypothesis formulation only.

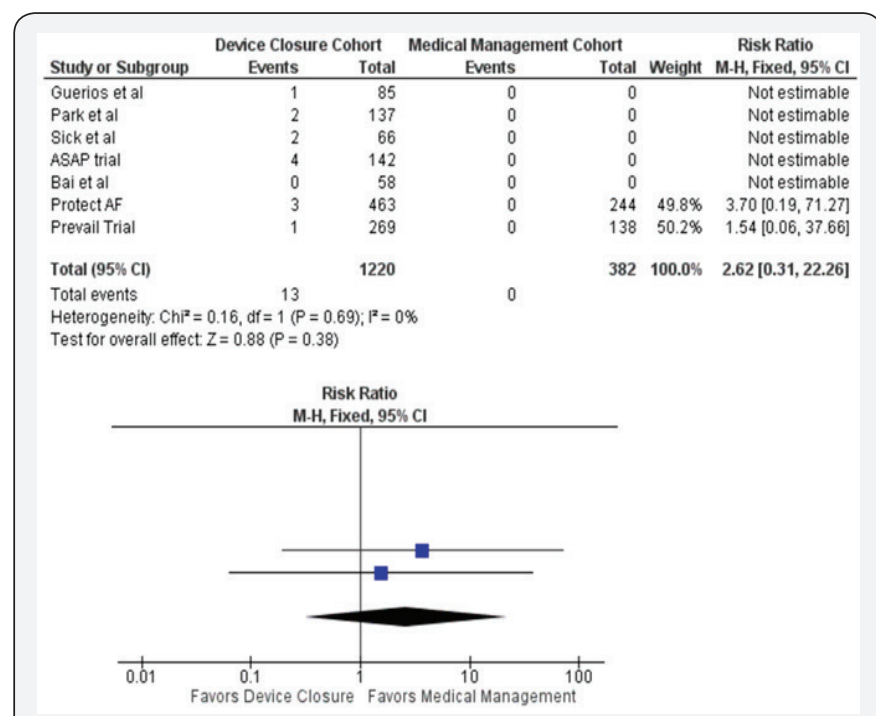

Figure 7: Systemic embolization incident rate- device based laa closure vs medical management.

Similar to the risk of hemorrhagic stroke, the risk of systemic embolization and overall mortality could only be compared in the PREVAIL and PROTECT AF trials, as these were the only trials with both a control and intervention group. Given the 
overall low power of the analysis of those outcomes (Figure 7 \& 8), neither endpoint reached statistical significance. The results of this analysis are appropriate for hypothesis formulation only, as residual confounding by indication cannot be avoided. While further research may continue to elucidate plausible mechanisms of benefit, large scale randomized trials designed a priori are necessary to test the hypothesis.

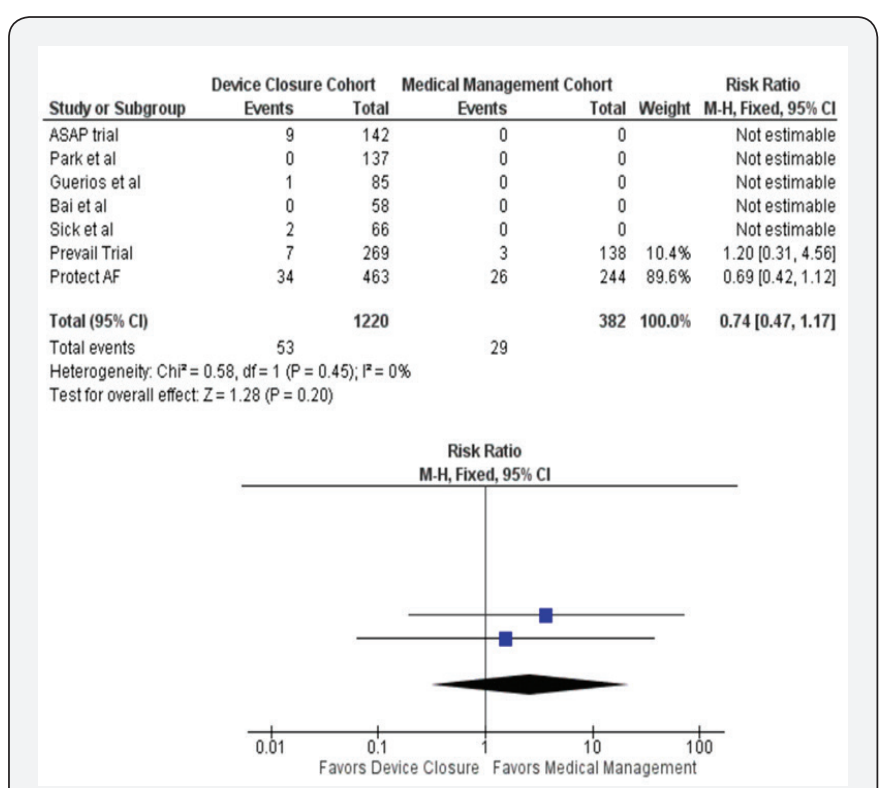

Figure 8: Death from all causes- device based laa closure vs medical management.

\section{Safety Outcomes}

\begin{tabular}{|c|c|c|c|c|c|c|c|c|c|}
\hline & \multicolumn{3}{|c|}{ Preval Trial ASAP Trial Protect AF Trial } & Baietal & Guerios etal & Parketal & Sicketal & Composite & \\
\hline Device Embolization & 2/269 & $2 / 150$ & 1/463 & 0/58 & 1/85 & 2/137 & 2/66 & $10 / 1228$ & $1 \%$ \\
\hline Pericardial Effusion with Tamponade & 1/269 & 2/150 & $8 / 463$ & $1 / 58$ & $1 / 85$ & $5 / 137$ & $1 / 66$ & $28 / 1228$ & $2 \%$ \\
\hline Major Bleeding Requiring Transtusion & 1/269 & 2/150 & NR & NR & NR & NR & NR & 3/419 & $1 \%$ \\
\hline Loss of Implant in venous system & $0 / 269$ & $0 / 150$ & 0/463 & $0 / 58$ & $0 / 85$ & 1/137 & $2 / 66$ & 3/1228 & $0.20 \%$ \\
\hline Air Embolism with transient ST elevation & NR & $\mathbb{N R}$ & 0/463 & NR & NR & 2/137 & 1/66 & 3/666 & $0.40 \%$ \\
\hline
\end{tabular}

Figure 9: Safety outcomes by trials.

As demonstrated in Figure 9, the overall incidence of procedure-related complications is rather low, with most outcomes occurring approximately $1-2 \%$ of the time. The most common complication of LAA closure device placement was pericardial effusion. Several of the centers participating in the included studies did not use intra-procedure echocardiography and implanted the device under fluoroscopic guidance only. This may, in part, explain the frequency of this particular adverse outcome. As per the protocol design, PREVAIL patients were at higher risk than PROTECT AF patients, with older age (74.0 \pm 7.4 years vs. $71.7 \pm 8.8$ years $[\mathrm{p}<0.001]$ ) and a higher CHADS2 score $(2.6 \pm 1.0$ vs. $2.2 \pm 1.2$ [p $<0.001]$ ). Compared with other trials, PREVAIL had more patients $>75$ years of age. PREVAIL patients also had a higher incidence of diabetes mellitus and a higher incidence of previous stroke/transient ischemic attack. Procedural and device-related strokes decreased, from $1.1 \%$ in PROTECT AF to $0.4 \%$ in PREVAIL ( $p<0.007$ ). Device embolization was infrequent, occurring in only 4 patients (2 in PROTECT AF, 2 in PREVAIL) and did not demonstrate statistically significant differences across the studies.

\section{Discussion}

The advent of LAA closure devices to treat patients with $\mathrm{AF}$ who are not candidates for chronic OAC offers an interesting and efficient option to the medical armamentarium. As the majority of the studies evaluating the outcomes with the LAA closure devices are observational series, and so few randomized trials are available in the literature, it is difficult to make head-tohead comparisons between groups. For this purpose, we used the CHADS2 score, which has been well validated and clinically used as predictor of ischemic embolic stroke in AF patients, including reliable stroke risk estimation per year. The results we obtained showed a significant difference in the composite endpoint of nonfatal stroke and TIA rate when compared to its respective CHADS2 score estimated risk on patients not treated with OAC. The absolute risk reduction was greater than $8 \%$ / year in patients with CHADS 2 score greater than 2 . For those individuals who will not be treated with OAC, the LAA closure is definitely an approach to consider.

As any intervention, the LAA closure procedure carries eventual intrinsic risk, with LAA perforation, pericardial hematoma and cardiac tamponade as its main complications. Our review demonstrated LAA closure carries an overall complication rate of $4.7 \%$, which we understand as being acceptable given the complexity of the procedure and the long term benefit provided after the device is successfully implanted. Pericardial hematomas leading to tamponade requiring emergent pericardiocentesis are the most concerning events. Nonetheless, once identified in timely manner and appropriately treated, the problem is solved and rarely requires further intervention or, even less commonly, leads to death. An interesting finding in PROTECT AF trial was the low rate of complication that was achieved by the involved operators, as low as 54 events per 980 patient years, or 5.5 events per 100 patient years with $95 \%$ CI of 4.2-7.1.

One point we believe our review contributes most is the analysis of the complication rate during the early years of LAA closure when compared to the results of the PROTECT AF trial. Pericardial hematoma and cardiac tamponade happened in $3.3 \%$ of cases in the initial series, where also death in the first 24 hours occurred in $1.1 \%$ of cases. In modern studies, those events were reduced to $0.7 \%$ and $1 \%$ respectively. Two main reasons may eventually explain the difference. First, the initial device (PLAATO) had a first generation design, which although efficient was more bulky, with 3 rows of anchors on a self-expandable nitinol cage with difficult trackability while positioning in the LAA. The Amplatzer and the Watchman devices are less bulky, but they also use anchors to attach in the LAA wall and stay fixed in position. This anchoring step during the procedure is where the risk for perforation reaches its peak, besides the 
"tag test" where confirmation of success anchoring is assessed. However, when analyzing devices isolated, our results did not show difference in the complication rate. Second and more importantly, the operators' learning curve may have played an important role in the early results when compared to the most recent PROTECT AF trial results, which is a reason why we excluded small studies with few patients enrolled. Development of better techniques and appropriate skills are most likely the factors that led to less complications in the recent trial. Additionally, most operators involved with PROTECT AF have been involved with the procedure since the early years and, therefore, have been accumulating experience and improving their patients' outcomes over time. The evolution of operator skills is particularly noteworthy, because as LAA closure becomes more popular and more centers and operators begin to offer this new technology, it must be kept in mind that the learning curve is steep and new and less experienced operators should exercise extreme caution as they proceed. Nonetheless, new operators should be excited, as LAA closure devices are a promising and evolving approach to helping people with $\mathrm{AF}$ who are unable to receive OAC.

Limitations of our study include those inherent to any retrospective analysis, specifically the absence of homogeneous randomized studies for comparison and the use of a fixed estimation score (CHADS2) to compare with actual events from the observational studies.

\section{Conclusion}

The transcutaneous LAA closure using an occlusion device is efficient to prevent stroke and TIA and safe when performed by experienced operators. New, less-experienced, operators should dedicate time to overcome the steep learning curve in order to achieve excellence.

\section{References}

1. Colilla S, Crow A, Petkun W, Singer DE, Simon T, et al. (2013) Estimates of current and future incidence and prevalence of atrial fibrillation in the US adult population. Am J Cardiol 112(8): 1142-1147.

2. Roger VL, Go AS, Lloyd-Jones DM, Adams RJ, Berry JD, et al. (2011) Heart disease and stroke statistics -2011 update: a report from the American Heart Association. Circulation 123(4): e18-e209.
3. Connolly SJ, Ezekowitz MD, Yusuf S, Phil D, John E, et al. (2009) Dabigatran versus warfarin in patients with atrial fibrillation. $\mathrm{N}$ Engl J Med 361: 1139-1151.

4. Connolly SJ, Eikelboom J, Joyner C, Eikelboom J, Oldgren J, et al. (2011) AVERROES Steering Committee and Investigators - Apixaban in patients with atrial fibrillation. N Engl J Med 361(12): 806-817.

5. Patel MR, Mahaffey KW, Garg J, Pan G, Singer DE, et al. (2011) ROCKET AF Investigators. Rivaroxaban vs. warfarin in nonvalvular atrial fibrillation. N Engl J Med 365(10): 883-891.

6. Granger CB, Alexander JH, McMurray JJ, Lopes RD, Hylek EM, et al. (2011) ARISTOTLE Committees and Investigators. Apixaban vs. warfarin in patients with atrial fibrillation. N Engl J Med 365(11): 981992.

7. Bungard TJ, Ghali WA, Teo KK, McAlister FA, Tsuyuki RT (2000) Why do patients with atrial fibrillation not receive warfarin? Arch Int Med 160(1): 41-46.

8. Nieuwlaat R, Capucci A, Camm AJ, Olsson SB, Andresen D, et al. (2005) Atrial fibrillation management: a prospective survey in ESC Member Countries: The Euro Heart Survey on Atrial Fibrillation. Eur Heart J 26(22): 2422-2434.

9. Landmesser U, Holmes DR (2012) Left atrial appendage closure: a percutaneous transcatheter approach for stroke prevention in atrial fibrillation. Eur Heart J 33(6): 698-704.

10. Blackshear JL, Odell JA (1996) Appendage obliteration to reduce stroke in cardiac surgical patients with atrial fibrillation. Ann Thorac Surg 61(2): 755-759.

11. Sievert H, Lesh MD, Trepels T, Omran H, Bartorelli A, et al. (2002) Percutaneous left atrial appendage transcatheter occlusion to prevent stroke in high risk patients with atrial fibrillation: early clinical experience. Circulation 105(16): 1887-1889.

12. Liberati A, Altman DG, Tetzlaff J, Mulrow C, Gøtzsche P, et al. (2009) The PRISMA statement for reporting systematic reviews and metaanalysis of studies that evaluate healthcare interventions: explanation and elaboration. BMJ 339: 2700.

13. Reddy VY, Doshi SK, Sievert H, Buchbinder M, Neuzil P, et al. (2013) Percutaneous Left Atrial Appendage Closure for Stroke Prophylaxis in Patients With Atrial Fibrillation: 2.3-Year Follow-up of the PROTECT AF (Watchman Left Atrial Appendage System for Embolic Protection in Patients With Atrial Fibrillation) Trial. Circulation 127(6): 720-729.

14. Bayard YL, Becht R, Ostermayer SH, Sievert H (2016) Left atrial appendage closure. Percutaneous interventions in congenital heart disease.

Commons Attribution 4.0 License

DOI: $10.19080 /$ JOCCT.2017.06.555689

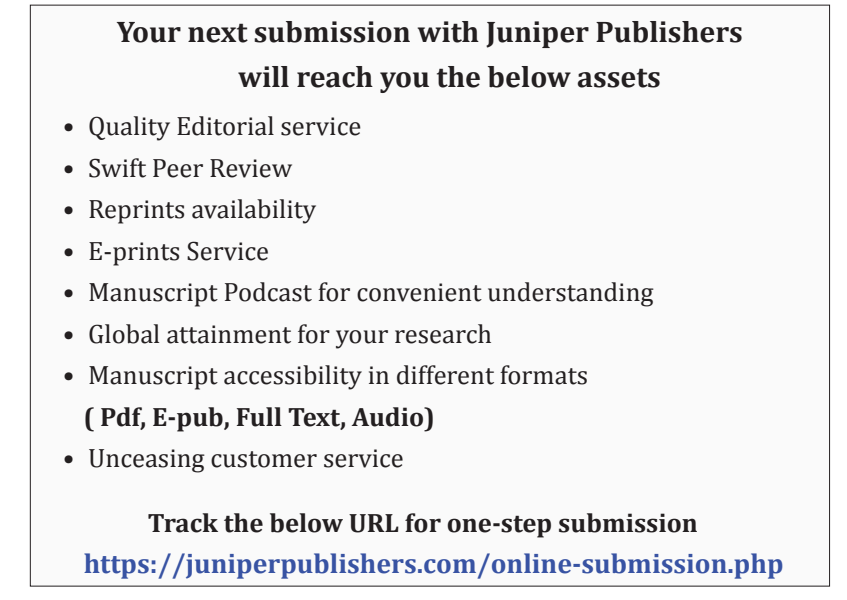

\section{Your next submission with Juniper Publishers} will reach you the below assets 